\title{
LA ANOSMIA Y SU INTERACCIÓN EN LAS INFECCIONES CAUSADAS POR EL SARS-COV2: UNA REVISIÓN
}

\section{Anosmia and its interaction in infections caused by SARS-CoV2: A review}

\section{Fernanda J. Rodríguez R}

Laboratorio de Errores Innatos del Metabolismo

Universidad Federal de Pará, Brasil.

Correo-e: fernandarodriguezramos@gmail.com

\section{Edian F. Franco}

Área de Ciencias Básicas y Ambientales, Instituto

Tecnológico de Santo Domingo (INTEC). Santo

Domingo, República Dominicana.

ORCID: 0000-0001-9715-9437

Correo-e: edianfranco@intec.edu.do

\section{Rommel T. Jucá Ramos}

Laboratório de Ingeniería Biológica, Universidad Federal de Pará, Brasil.

ORCID: 0000-0002-8032-1474

Correo-e: rommelthiago@gmail.com

Maria H. Thomaz Maia

Laboratorio de Errores Innatos del Metabolismo,

Universidad Federal de Pará, Brasil.

Correo-e: mariahtmaia@gmail.com

Recibido: 23/11/2021 • Aprobado: 15/12/2021

Cómo citar: Franco, E. F., Rodríguez R, F. J., Jucá Ramos, R. T., \& Thomaz Maia, M. H. (2021). La anosmia y su interacción en las infecciones causadas por el SARS-CoV2: una revisión. Ciencia, Ambiente y Clima, 4(2), 7-17. Doi: https://doi.org/10.22206/cac.2021.v4i2.pp7-17

\section{Resumen}

La anosmia es uno de los síntomas frecuentes identificados como parte del cuadro clínico de la infección por SARS-CoV2, sin embargo, los mecanismos por los cuales ocurre la disfunción olfatoria no están completamente esclarecidos. Previamente los coronavirus fueron identificados como una familia de virus que pueden causar anosmia, pero en el COVID-19, a diferencia de la familia de los rinovirus, esta no se relaciona con un proceso inflamatorio de la mucosa nasal asociado a obstrucción nasal o rinorrea, lo cual sugiere que la anosmia podría deberse a disturbios en la conducción nerviosa del estímulo olfatorio. La anosmia de los pacientes con COVID-19 recuerda en algunos aspectos al síndrome de Kallman, el cual también cursa con anosmia, su etiología es genética y se debe a mutaciones en el gen ANOS 1 que codifica la molécula de adhesión celular anosmia. En esta revisión se suministrará una recopilación de evidencias fisiopatológicas e interacciones moleculares mediante la cual la proteína anosmia podría modificar el curso de la infección por SARS-CoV2, para ello, se realizó una búsqueda computarizada de literatura para desvendar las posibles relaciones que existen en entre la anosmia y las infecciones causadas por el SARS-CoV2.

Palabras clave: SARS-CoV-2; anosmia; COVID-19; revisión; interacción.

\section{Abstract}

Anosmia is one of the frequent symptoms identified as part of the clinical picture of SARS-CoV2 infection. However, the mechanisms by which olfactory dysfunction occurs are not entirely clarified. Previously, coronaviruses were identified as a family of viruses that can cause anosmia. However, in COVID-19, unlike the rhinovirus family, this is not related to an inflammatory process of the nasal mucosa associated with nasal obstruction or rhinorrhea, which suggests that the anosmia could be due to disturbances in the nerve conduction of the olfactory stimulus. The anosmia of patients with COVID-19 is reminiscent of some aspects of Kallman's syndrome, which also occurs with anosmia; its etiology is genetic and is due to mutations in the ANOS 1 gene that encodes the cell adhesion molecule anosmia. This review will provide a compilation of pathophysiological evidence and molecular interactions through which the anosmia protein could modify the course of the SARS-CoV2 infection. For this, a computerized literature search was carried out to unravel the possible relationships between anosmia and infections caused by SARS-CoV-2.

Keywords: SARS-CoV-2, Anosmia, COVID-19, review, interaction. 


\section{Introducción}

Las evidencias actuales demuestran que los principales síntomas asociados al COVID-19 no se limitan al distrés respiratorio, siendo reportados otros signos y síntomas relacionados a la invasión del virus al sistema neurológico, entre estos se encuentra la cefalea, náuseas y vómitos. Uno de los síntomas frecuentemente identificado es la anosmia y la ageusia, cuya fisiopatología aún no está bien esclarecida, en cuanto a si se trata de una lesión local en el epitelio neurolfatorio o por compromiso de las neuronas del bulbo olfatorio (Brann et al., 2020; Lechien et al., 2020; Li, Bai, \& Hashikawa, 2020a; Russell et al., 2020a; Townend et al., 2018).

La anosmia es definida como la imposibilidad para detectar cualitativamente las sensaciones olfatorias, es decir, ausencia de función olfatoria (anosmia total), o habilidad para percibir solo algunos odorantes (anosmia parcial). Puede ser causada por un traumatismo craneal, daño permanente en el neuroepitelio olfatorio, infecciones respiratorias o tener un origen congénito, mientras que la hiposmia o microsmia, es solo la disminución sensibilidad a para la detección de olores. La capacidad olfativa no es constante a lo largo de la vida, su agudeza máxima se presenta entre la tercera y quinta décadas y disminuye notablemente después de la séptima hasta unas tres cuartas partes a los 80 años. A partir de los 65 años, debido a la disminución del número de neuronas sensoriales receptivas de olores, adelgazamiento del epitelio, alteraciones en las células olfatorias receptivas, reemplazo del epitelio olfatorio por epitelio respiratorio, en algunos casos la anosmia se presenta como un síntoma temprano de enfermedades neurodegenerativas como Parkinson, Alzheimer y cuando se manifiesta en el deterioro cognitivo leve, puede anunciar la progresión a la demencia (Doty et al., 1984; Fuentes et al., 2011).

El deterioro quimiosensorial caracterizado por anosmia y ageusia han sido reportados en 30 a $68 \%$ de los pacientes con infección con COVID-19, el reco- nocimiento de la anosmia como un síntoma de COVID-19 podría alentar a más personas a autoaislarse, incluso en ausencia de otros síntomas, para evitar una mayor propagación del virus, además de ayudar a la comprensión de la fisiopatología de la enfermedad y también como factor pronóstico (Russell et al., 2020b; Yan, Faraji, Prajapati, Boone \& DeConde, 2020)

Previamente los coronavirus fueron identificados como una familia de virus que pueden causar anosmia, pero en el COVID-19, a diferencia de la familia de los rinovirus, esta no se relaciona con un proceso inflamatorio de la mucosa nasal, con consecuente rinorrea como ocurre en infecciones por rinovirus, parainfluenza y Epstein Barr, así lo demuestra un estudio donde el 79,7\% de los pacientes con diagnóstico confirmado de COVID-19 presentaron disfunción olfatoria en ausencia de obstrucción nasal o rinorrea (Lechien et al., 2020; Yan et al., 2020); esto sugiere que la anosmia podría deberse a disturbios en la conducción nerviosa del estímulo olfatorio, probablemente por interferencia de la diseminación axonal del virus desde el epitelio olfatorio nasal hasta el sistema nervioso central, considerando que la infección por SARS-CoV-2 ha sido reportada en el cerebro tanto de pacientes como de animales experimentales, con fuerte afectación del tronco encefálico (Dubé et al., 2018; Li, Bai, \& Hashikawa, 2020b).

Esta revisión tiene por objetivo recopilar y analizar las evidencias fisiopatológicas e interacciones moleculares mediante la cual la proteína anosmia podría modificar el curso de la infección por SARS-CoV2.

\section{Anatomía y fisiología del olfato}

El epitelio nasal se divide anatómicamente en un epitelio respiratorio (RE) y un epitelio olfativo sensorial (OE), en la cual el RE nasal se caracteriza por ser continuo con el epitelio que recubre gran parte del tracto respiratorio y humedece el aire que ingresa por la nariz; se conforma de células basales, 
las células ciliadas, las células secretoras (incluidas las células caliciformes) y las células de ciliadas (microvellosidades) mientras que el $\mathrm{OE}$ es una estructura laminada que alberga neuronas sensoriales olfativas maduras (OSN), estas neuronas receptoras olfativas son células bipolares embriológicamente derivadas tanto del placoda olfatorio como de la cresta neural $\mathrm{y}$ forman grupos dentro del neuroepitelio respiratorio. Contienen 3-50 cilios en los que se encuentran los receptores de olor y envían sus axones no mielinizados a través de la placa cribiforme para hacer sinapsis en el bulbo olfatorio. Los circuitos locales del bulbo olfatorio, procesan la información olfatoria antes de enviarla hacia los centros cerebrales superiores (Attems, Walker, \& Jellinger, 2015; Brann et al., 2020; Smith \& Bhatnagar, 2019).

Los odorantes se unen a los receptores de unión a nucleótidos de guanidina acoplados a proteínas de unión a $(\mathrm{G})$ codificados por más de 500 de genes, pero solo de 100 a 200 receptores son funcionales en los cilios de las neuronas receptoras olfativas. La transducción de señales está mediada por la activación de la adenil ciclasa 3, con monofosfato de adenosina cíclico 3', 5' (AMPc) que desencadena la apertura de canales cloro activados por calcio que generan despolarización, la cual es amplificada por el flujo de cloruro de las neuronas sensoriales (Attems et al., 2015). El bulbo olfatorio es el primer sitio de procesamiento de la información olfatoria en el cerebro, se localiza bilateralmente por encima de la placa cribiforme de la base anterior del cráneo cuyas perforaciones dejan pasar ramificaciones del nervio olfatorio. Con un volumen promedio en humano de 125-17 mm3 (Dammalli et al., 2017). El $81 \%$ de las proteínas expresadas en el bulbo olfatorio están asociadas al transporte de moléculas.

\section{Interacción de los coronavirus con el sistema olfatorio}

En pacientes con COVID-19 confirmado y anosmia, mediante resonancia magnética, se evidenció que el bulbo y tracto olfatorio presentaba volumen e inten- sidad de señal normal sin signos de congestión nasal (Galougahi et al., 2020). La presencia de proteína viral de otro tipo de coronavirus ( $\mathrm{HCoV}$ OC43) ha sido observada en el bulbo olfatorio de ratones C57BL/6 al tercer día después de la inoculación intranasal del virus (Dubé et al., 2018). Sin embargo, mediante comparación de datos de RNA -Seq y scSeq del sistema olfatorio se evidenció que solo los pericitos y no las células neurales, madres y perivasculares del bulbo olfatorio expresan el receptor más reconocido (ACE2) y proteasas (TMPRSS2, Catepsina B y L, CTSB/CTSL) que facilitan la entrada del CoV2 al interior de la célula, en tal sentido, sería lógico pensar que podrían existir otros receptores mediante los cuales el virus logre ingresar al sistema nervioso central, pues la difusión pasiva de las partículas virales y el transporte axonal son estrategias de propagación válidas utilizadas por otros coronavirus. La expresión de marcadores de diferentes clases celulares y genes que codifican proteínas de entrada del cov-2 en bulbo olfativo de ratón, demuestran que las más expresas son Ace2 en células vasculares, Ctsb en células inmonológicas, Ctsl en inmunológica y astrocitos; otros CoV: Bsg (células vasculares), Hspa5 en células precursoras de oligodendrocitos, células inmunológicas, neuronas, astrocitos y células precursoras intermedias (Brann et al., 2020; Dubé et al., 2018).

La proteína Basigin codificada por el gen Bsg expresado en células vasculares infectadas por otros coronavirus, juega un papel importante en la orientación de los transportadores de monocarboxilato SLC16A1, SLC16A3, SLC16A8, SLC16A11 y SLC16A12 a la membrana plasmática; desempeña funciones fundamentales en la espermatogénesis, la implantación de embriones, la formación de redes neuronales y la progresión tumoral. Estimula los fibroblastos adyacentes para producir metaloproteinasas de matriz. Parece ser un receptor para los glicanos oligomannosídicos. In vitro, promueve el crecimiento de procesos astrocíticos, induce la producción de citosinas proinflamatorias con la IL-6 en monocitos (Bateman, Wu, \& Bridge, 2020; Brann et al., 2020). 
Contradictoriamente, datos de scRNA-seq obtenidos de un Sistema de regeneración in vitro de células epiteliales nasales, corroboraron las expresión de $A C E 2$ en las células globosas/secretoras y células ciliadas en los cultivos de interfase aire-liquído, además, se observó un aumento progresivo de ACE2, lo cual podría ser relevante desde el punto de vista de la patogénesis; vale la pena señalar que TMPRSS2 solo se expresó en un subconjunto de Células ACE2+, lo que insinúa que el virus podría usar caminos alternativos como las proteasas catepsina B/L. Simultáneamente, en ese estudio se demostró la correlación de la expresión aleatoria entre la ACE2 en las vías aéreas superiores y genes asociados al metabolismo de los carbohidratos y al sistema inmunológico, entre ellos el IDO1, IRAK3, NOS2, TNFSF10, $O A S 1$ y $M X 1$, los cuales tuvieron una mayor expresión en las células globosas nasales 1 y 2, además de las células nasales ciliadas. La expresión de estos genes relacionados con el sistema inmunológico estaría asociada a una susceptibilidad menor a la infección viral (Sungnak et al., 2020).

\section{Probable fisiopatología de la anosmia durante la infección por SARS-CoV-2}

Uno de los modelos propuestos para explicar el déficit quimio sensorial, es que el SARS-CoV2 se dirige al epitelio olfativo, el cual puede regenerarse y repararse rápidamente después del aclaramiento viral, la expresión de ACE2 en las células sustentaculares y basales del epitelio olfatorio y su ausencia en las neuronas olfativas sensoriales, sugieren que la infección de subconjuntos de células sustentaculares puede ser suficiente para causar un cascada fisiopatológica que culmina en daños a las OSN y defectos en la función olfatoria (Brann et al., 2020; Hu, Guo, Zhou, \& Shi, 2021; Yan et al., 2020).

El suministro intranasal (IN) de células madres mesenquimales y células de glioma al cerebro demuestra que las células aplicadas por vía intranasal pueden migrar al cerebro intacto a través de la placa cribiforme a lo largo de la vía neural olfatoria y posi- blemente a lo largo de otras rutas de migración. El pretratamiento intranasal con hialuronidasa mejora la entrega de células madre mesenquimales al cerebro. El hecho de que las células aplicadas con IN puedan viajar a través de la placa cribiforme es consistente con varios informes que muestran que, durante el desarrollo prenatal, las neuronas que secretan la hormona liberadora de gonadotropina migran desde el compartimento nasal como el sitio de su origen a través de la placa cribiforme hacia el cerebro anterior (Danielyan et al., 2009).

Los pacientes con COVID 19 y disfunción quimio sensorial relatan que la disfunción olfatoria apareció antes (11.8\%), después (65.4\%) o al mismo tiempo que la aparición de síntomas generales o en oído, nariz y garganta $(22.8 \%)$, inclusive, el porcentaje de pacientes que presentaron afectación olfatoria después de los síntomas sería mayor, teniendo en cuenta que el 9,4\% de los pacientes no recordaba el momento de inicio de la disfunción olfatoria (Gupta et al., 2020; Hu et al., 2021). La propagación del CoV-2 a través del epitelio respiratorio depende de la presencia de principalmente de los receptores ACE2 y proteasas de superficie como la TMPRSS2, siendo más rápida que la difusión axonal y pasiva del virus hacia el SNC vía olfatoria, explicando probablemente la secuencia de aparición de los síntomas (Dubé et al., 2018; Huang et al., 2021; Netland, Meyerholz, Moore, Cassell, \& Perlman, 2008). La disfunción quimiosensorial podría coincidir entonces con la apoptosis inducida de las neuronas sensoriales olfatorias después de una lesión a nivel del bulbo olfatorio generada por la difusión hacia el SNC. Esta degeneración neuronal masiva, que ocurriría durante los primeros cinco días después de la infección recuerda a los mecanismos de muerte neuronal asociados a lesiones del bulbo olfatorio. En modelos experimentales en la cual se lesiona el bulbo olfatorio, se observa una reducción dramática (50-70\%) en el grosor del epitelio olfativo posterior del bulbo olfatorio, al mismo tiempo, se observa un aumento de la proliferación de células precursoras 
neuronales en el compartimento basal del neuroepitelio. La respuesta proliferativa, que alcanza un pico a los cinco días después de la lesión, da como resultado el reemplazo de muchas de las neuronas perdidas (Huang et al., 2020; Shovlin \& Tropea, 2018; Vaira, Salzano, Fois, Piombino, \& De Riu, 2020; Wang et al., 2020).

En el mismo orden de ideas, la recuperación en caso de anosmia los pacientes con hiposmia o anosmia tuvieron los siguientes tiempos de recuperación: $1-4$ días (33.0\%), 5-8 días (39.6\%), 9-14 días (24.2\%) y más de 15 días $(3.3 \%)$, aunque hay pacientes que han presentado disfunciones olfativas hasta seis meses después de la infección (Lechien et al., 2021; Marinosci, Landis, \& Calmy, 2020), la fase de recuperación quimiosensorial nuevamente coincidirá con la reconstitución del epitelio olfatorio después de lesiónes del bulbo olfatorio; se han observado diferencias en la reconstitución del epitelio olfativo según la presencia o ausencia del objetivo sináptico, el bulbo olfatorio. La restauración del epitelio olfativo después de la axotomía es consistentemente mejor que después de la bulbectomía, con recuperación a valores cercanos al control después de 30 días después de la lesión, ese periodo podría ser menor si la lesión fuese transitoria como estamos sugiriendo en el caso de COVID-19. Por el contrario, incluso a largos intervalos después de una bulbectomía unilateral (1-9 meses), el epitelio olfativo reconstituido no ha recuperado completamente el grosor del lado de control. Además, el epitelio olfativo en el lado lesionado permanece en un estado relativamente inmaduro. Varias investigaciones han demostrado que el epitelio reconstituido exhibe una regulación positiva de la proliferación de precursores neuronales que se acompaña de un aumento en la tasa de muerte celular, en ausencia del tejido objetivo, la vida útil de las neuronas recién generadas se acorta notablemente, del orden de 10 días o menos, lo que sugiere una dependencia trófica de las neuronas olfativas del bulbo olfatorio para su supervivencia prolongada (Chen et al., 2021; Gupta et al., 2020; Shovlin \& Tropea, 2018; Vaira et al., 2020),
A pesar que las células neurales del bulbo olfatorio no expresan los receptores y proteasas para el ingreso de las células, mediante tinción inmunohistoquímica del tejido de biopsia del epitelio olfativo humano se identificó que las células madres olfatorias y células de sustentación olfatorias si expresan tales elementos, pudiendo explicar la anosmia debido a la modificación de la estructura del epitelio olfatorio por la afectación de las células de sustentación que dan soporte a las OSNs, las cuales detectan los odorantes a través de sus cilios dendríticos (Brann et al., 2020; Gupta et al., 2020). Sin embargo, las células basales globosas (GBC) son las principales responsables de la regeneración de las OSN durante el recambio epitelial normal, y las células basales horizontales (HBC) actúan como células madre de reserva activadas por el daño tisular todos los subtipos de HBC expresaron ACE2, la frecuencia de expresión de ACE2 fue menor en los HBC olfativos $(0,84 \%$ de las células) en comparación con los $\mathrm{HBC}$ respiratorios (1,78 \% de las células) (Brann et al., 2020; Gupta et al., 2020)

\section{Anosmia}

La anosmia de los pacientes con COVID-19 nos recuerda en algunos aspectos al sindrome de Kallman (MIM 300836, MIM 604846) caracterizado clínicamente por anosmia e Hipogonadismo hipogonadotrófico, su etiología es genética, se debe a mutaciones en el gen ANOS 1 (Anosmin 1 o Kal-1) localizado en el Xp22.31, confiriéndole una herencia recesiva ligada al cromosoma $\mathrm{X}$, siendo por lo tanto más frecuente en el sexo masculino, aunque también ha sido descrita una forma autosómica dominante con o sin anosmia asociada a mutaciones en el gen HS6ST1 (Heparan sulfate 6-o-sulfotransferase 1), locus 2q14.3. El Kal-1 / Anosmin-1, codifica una molécula de adhesión celular (la anosmina), cuando muta causa focalización neuronal y defectos de migración de algunos tipos celulares entre ellas las neuronas que secretan la hormona liberadora de gonodatrofinas (Hormona folículo estimulante: FSH y Hormona luteinizante: $\mathrm{LH})$, el fenotipo migratorio de esos tipo celulares es dependiente de la dosis de anosmia. 
Además del sistema olfativo principal existe un sistema quimiosensorial separado presente en todas las especies de vertebrados, incluido el hombre, llamado nervio terminal o nervus terminalis, este nervio se ramifica por todo el epitelio nasal antes de cruzar la mucosa nasal y atravesar los agujeros de la placa cribiforme medial hasta el nervio olfatorio; las neuronas del nervio terminal derivan del plexo submucoso del tabique nasal y tanto los somas como los axones del $70 \%$ de todas las neuronas del nervio terminal son inmunorreactivas para la liberación de la hormona luteinizante hormona (LHRH). El nervio terminal se proyecta directamente desde la nariz hasta regiones específicas del cerebro anterior (incluyendo los hemisferios, tálamo e hipotálamo) involucradas con la recepción y coordinación de los estímulos que evocan un determinado comportamiento y cambios hormonales relevantes para la reproducción. Las hormonas sexuales, hormona liberadora de hormonas luteinizantes (LHRH) y estrógeno, funcionan como neurotransmisores en áreas de procesamiento olfativo. (Stockhorst \& Pietrowsky, 2004)

\section{Anosmia y el SARS-CoV-2}

La evidencia emergente sugiere que mueren más hombres que mujeres, posiblemente debido a diferencias inmunológicas de género. Sin embargo, los datos desglosados por sexo actuales son incompletos y no se pueden llegar a conclusiones. La posibilidad de un efecto hormonal tampoco puede excluirse, las evidencias muestran que las vías de señalización estrogénicas juegan un papel crítico en la patogénesis de enfermedades con afectación pulmonar mediada por los recetores estrogénicos Era y ERb, los cuales están ampliamente expresados en el tejido pulmonar, además se conoce que el estradiol aumenta el ADAM17 (Tumor Necrosis Factor- Convertase) y este a su vez es responsable de la proteasa para la eliminación de la ACE2 (receptor utilizado por el CoV-2) (Chen et al., 2021; Colangelo et al., 2014; Gupta et al., 2020; Lambert et al., 2005; Ren et al., 2015; Wenham, Smith, \& Morgan, 2020).
Los experimentos in-vitro e in-vivo sugieren que KAL-1 / anosmina-1 desempeńa un papel de co-factor autocrino en la ramificación dendrítica formando un complejo con el receptor de crecimiento de fibroblasto EGL-15 / FGFR a través de la molécula de adhesión celular SAX-7/L1CAM, a su vez el EGL-15 / FGFR requiere de la señalización Ras aguas abajo. En el mismo orden de ideas, la SAX-7 es una proteína transmembrana de un solo paso con un dominio de inmunoglobulina extracelular (Ig) y dominios FN (III) (Díaz-Balzac, Lázaro-Peña, Ramos-Ortiz, \& Bülow, 2015).

El dominio intracelular de la molécula de adhesión celular SAX-7 / L1CAM, es prescindible para la ramificación de neuritas, pero no para el posicionamiento celular pudiendo proporcionar especificidad espacial a través de la localización subcelular a los complejos resultantes con otras moléculas, mientras que SAX-7 / L1CAM puede proporcionar especificidad a dichos complejos de SAX-7 / L1CAM. La función FGFR es esencial para el desarrollo de tejidos no neuronales y neuronales, estudios en ratones han demostrado que la señalización de FGF8 a través del FGFR1 es necesaria para la neurogénesis y la morfogénesis del epitelio olfativo (Díaz-Balzac et al., 2015).

Mediante clonación y expresión de 26 de las 29 proteínas virales en células humanas se identificaron las proteínas humanas físicamente asociadas con cada una utilizando la purificación por afinidad mediante espectrometría de masas, un total de 332 interacciones proteína-proteína SARS-CoV-2-humano de alta confianza fueron identificadas, entre ellas la FGFR1 oncogene partner (FGFR1OP), Ras GTPase-activating protein-binding protein 1 (G3BP1), Ras GTPase-activating protein-binding protein 2 (G3BP-2), Ras-related protein Ral-A (RALA), Ras-related protein Rab-5C (RAB5L), Ras-related protein Rab-7a (RAB7A), Ras-related protein Rab-8A (RAB8A), Ras-related protein Rab-2A (RAB2A), Ras-related protein Rab-10 (RAB10), Ras-related protein Rab-14 (RAB14), Ras-related protein Rab-1A (YPT1-related protein/ RAB1A), 
Heparan sulfate 2-O-sulfotransferase 1 (HS2ST1), Ras-related protein Rab-18 (RAB18), Heparan-sulfate 6-O-sulfotransferase 2 (HS6ST-2) (Gordon et al., 2020; Zhou, Bao, \& Ning, 2021).

La heparán-6O-sulfotransferasa, enzima también involucrada en la fisiopatología del síndrome de Kallman, se localizan en el aparato de Golgi y cataliza la transferencia de un residuo de sulfato en la posición 6 del residuo de glucosamina, que forma parte de la unidad de repetición disacárida característica de los sulfatos de heparán, que son componentes integrales de los proteoglicanos de sulfato de heparán. Esta enzima depende de la actividad de la anosmia y su actividad puede inhibirse por la heparina o por altas concentraciones de sal, no obstante, la capacidad inhibitoria de la heparina se anula por alteraciones en el dominio WAP. La anosmia media la ramificación dendrítica como un cofactor autocrino con el EGL-17 / FGF (factor de crecimiento de fibroblastos) a través de un complejo receptor que consiste en la molécula de adhesión celular conservada SAX-7 / L1CAM y el receptor del factor de crecimiento de fibroblastos EGL-15 / FGFR. Este complejo de proteínas, que parece conservado en humanos, requiere los dominios de inmunoglobulina (Ig) de SAX-7 / L1CAM y los dominios FN (III) de KAL-1 / anosmina-1 para la formación in-vitro y la función in-vivo (Fechner, Fong, \& McGovern, 2008; Oliveira et al., 2001).

Las L1CAMS son moléculas de adhesión celular conservadas en Caenorhabditis elegans, Drosophila melanogaster y vertebrados, que son importantes para el desarrollo y la función del sistema nervioso. Las L1CAM comparten un plan corporal básico de seis dominios de tipo inmunoglobulina y cinco repeticiones de fibronectina tipo III en el dominio extracelular, un dominio transmembrana único y una cola citoplasmática altamente conservada. Estos dominios promueven interacciones homofílicas y heterofílicas con diversas moléculas para promover la adhesión celular y la regulación de la transducción de señales en procesos que incluyen guía axonal, mielinización, fasciculación y mantenimiento de la arquitectura neural, así como la formación y mantenimiento de sinapsis (Opperman et al., 2014).

La vía de señalización de Notch juega un papel importante en el control del destino celular durante el desarrollo y en la vida postnatal y ha sido propuesta como responsable del mantenimiento de la homeostasis del sistema cardiovascular y mediador de las complicaciones cardiovascular como miocarditis, paro e insuficiencia cardíacos aguda durante la infección por COVID-19. En humanos, hay cuatro receptores (Notch1-4) activados por la unión con ligandos (Jagged1, 2 y ligandos similares a Delta $1,3,4)$ en la superficie de las células adyacentes. El precursor Notch es escindido primero por la furina en el aparato de Golgi y luego se encuentra como un heterodímero en la membrana celular. Después de la unión al ligando, es escindido por ADAM10 o ADAM17 en la membrana celular, permitiendo así la escisión final por la $\gamma$-secretasa que libera el dominio intracelular activo de Notch que migra al núcleo y regula la transcripción de genes diana. El ADAM 10 participa en la escisión de la molécula de adhesión L1 en la superficie celular y en las vesículas de membrana liberadas, lo que sugiere una actividad proteasa basada en vesículas; por lo tanto, la anosmia también participaría indirectamente en la vía de señalización Notch a través de la formación de complejos con la LICAM y podría estar compitiendo por este, evitando así la formación de complejos EGL-17 / FGF (facto de crecimiento de fibroblastos) - SAX-7 / L1CAM y los dominios FN (III) de KAL-1 / anosmina-1 necesario llevar a cabo su función colaborando así en la etiología del COVID-19 y se plantearía como un posible modulador del ingreso celular del CoV-2 a la célula y blanco terapeútico (Rizzo et al., 2020). La expresión de la anosmia no es constante a lo largo de la vida, siendo aparentemente mayor en mayores de 65 años y su expresión ha sido confirmada en 
tejidos alvos del Cov-2 como el pulmonar y SNC. (Bateman, Wu, \& Bridge, s. f.).

\section{Conclusión}

Este trabajo hace una revisión de los factores y características de la anosmia, en las infecciones causadas por SARS-CoV2, donde fueron abordados los principales mecanismos de interacción del virus con el sistema olfativo, así como la probable fisiopatología de la anosmia durante el desarrollo de la infección. Por otro lado, fueron abordados los diferentes factores genéticos y proceso neuronales que pueden estar involucrado en el desarrollo de este síntoma de la infección por el virus, el cual se convirtió en una de las manifestaciones más evidente para los pacientes infectados por este virus y que puede dejar secuelas que pueden persistir hasta seis meses después de la infección.

\section{Agradecimientos}

Este artículo fue parte de los últimos trabajos desarrollados por Fernanda J. Rodríguez R, la cual falleció en Brasil en mayo de 2021 a causa del SARS-CoV2. La publicación de esta revisión es un reconocimiento y homenaje a la labor de esta joven investigadora.

El presente trabajo fue desarrollado con el apoyo del Ministerio de Educación Superior Ciencia y Tecnología de la República Dominicana a través del proyecto: Mapeo de resistomas en los ríos Yaque del Norte y Yaque del Sur y sus implicaciones para la salud humana (Cod. 2018-2019-2B4-157).

\section{Referencias}

Attems, J., Walker, L., \& Jellinger, K. A. (2015). Olfaction and Aging: A Mini-Review. Gerontology, 61(6), 485-490. doi: 10.1159/000381619

Bateman, A., Wu, C. H., \& Bridge, A. J. (s. f.). (UniProt), The Universal Protein Resource.

Bateman, A., Wu, C. H., \& Bridge, A. J. (2020). The Universal Protein Resource (UniProt).

Brann, D. H., Tsukahara, T., Weinreb, C., Lipovsek, M., Van den Berge, K., Gong, B., ...
Datta, S. R. (2020). Non-neuronal expression of SARS-CoV-2 entry genes in the olfactory system suggests mechanisms underlying COVID-19-associated anosmia. bioRxiv, 2020.03.25.009084. Doi: 10.1101/2020.03. 25.009084

Chen, X., Laurent, S., Onur, O. A., Kleineberg, N. N., Fink, G. R., Schweitzer, F., \& Warnke, C. (2021, vasario 1). A systematic review of neurological symptoms and complications of COVID-19. Journal of Neurology, T. 268, 392-402. Springer Science and Business Media Deutschland GmbH. Doi: 10.1007/s00415020-10067-3

Colangelo, V., François, S., Soldà, G., Picco, R., Roma, F., Ginelli, E., \& Meneveri, R. (2014). Next-generation sequencing analysis of miRNA expression in control and FSHD myogenesis. PloS one, 9(10), e108411. Doi: 10.1371/journal.pone.0108411

Dammalli, M., Dey, G., Madugundu, A. K., Kumar, M., Rodrigues, B., Gowda, H., ... Prasad, T. S. K. (2017). Proteomic Analysis of the Human Olfactory Bulb. OMICS A Journal of Integrative Biology, 21(8), 440-453. Doi: 10.1089/omi.2017.0084

Danielyan, L., Schäfer, R., von Ameln-Mayerhofer, A., Buadze, M., Geisler, J., Klopfer, T., ... Frey, W. H. (2009). Intranasal delivery of cells to the brain. European Journal of Cell Biology, 88(6), 315-324. Doi: 10.1016/j. ejcb.2009.02.001

Díaz-Balzac, C. A., Lázaro-Peña, M. I., RamosOrtiz, G. A., \& Bülow, H. E. (2015). The Adhesion Molecule KAL-1/anosmin-1 Regulates Neurite Branching through a SAX-7/ L1CAM-EGL-15/FGFR Receptor Complex. CellReports, 11(9), 1377-1384. Doi: 10.1016/ j.celrep.2015.04.057

Doty, R. L., Shaman, P., Applebaum, S. L., Giberson, R., Siksorski, L., \& Rosenberg, L. (1984). Smell identification ability: Changes with age. Science, 226(4681), 1441-1443. Doi: 10. $1126 /$ science. 6505700 
Dubé, M., Le Coupanec, A., Wong, A. H. M., Rini, J. M., Desforges, M., \& Talbot, P. J. (2018). Axonal Transport Enables Neuron-toNeuron Propagation of Human Coronavirus OC43. Journal of Virology, 92(17), 1-21. Doi: 10.1128/jvi.00404-18

Fechner, A., Fong, S., \& McGovern, P. (2008, kovo). A review of Kallmann syndrome: Genetics, pathophysiology, and clinical management. Obstetrical and Gynecological Survey, T. 63, 189-194. Doi: 10.1097/OGX.0b013e31816 41278

Fuentes, A., Fresno, M. J., Santander, H., Valenzuela, S., Gutiérrez, M. F., \& Miralles, R. (2011). Sensopercepción olfatoria: Una revisión. Revista Medica de Chile, 139(3), 362-367. Doi: 10.4067/S0034-98872011000300013

Gordon, D. E., Jang, G. M., Bouhaddou, M., Xu, J., Obernier, K., White, K. M., ... Krogan, N. J. (2020). A SARS-CoV-2 protein interaction map reveals targets for drug repurposing. Nature 2020 583:7816, 583(7816), 459-468. Doi: 10.1038/s41586-020-2286-9

Gupta, A., Madhavan, M. V., Sehgal, K., Nair, N., Mahajan, S., Sehrawat, T. S., ... Landry, D. W. (2020, liepos 10). Extrapulmonary manifestations of COVID-19. Nature Medicine, T. 26, 1017-1032. Nature Publishing Group. Doi: 10.1038/s41591-020-0968-3

Hu, B., Guo, H., Zhou, P., \& Shi, Z. L. (2021, spalio 6). Characteristics of SARS-CoV-2 and COVID-19. Nature Reviews Microbiology, T. 19, 141-154. Nature Publishing Group. Doi: 10.1038/s41579-020-00459-7

Huang, C., Wang, Y., Li, X., Ren, L., Zhao, J., Hu, Y., ... Cao, B. (2020). Clinical features of patients infected with 2019 novel coronavirus in Wuhan, China. The Lancet, 395(10223), 497-506. Doi: 10.1016/S0140-6736(20)30183-5

Huang, N., Pérez, P., Kato, T., Mikami, Y., Okuda, K., Gilmore, R. C., ... Byrd, K. M. (2021). SARS-CoV-2 infection of the oral cavity and saliva. Nature Medicine, 27(5), 892-903. Doi: 10.1038/s41591-021-01296-8
Lambert, D. W., Yarski, M., Warner, F. J., Thornhill, P., Parkin, E. T., Smith, A. I., ... Turner, A. J. (2005). Tumor necrosis factor- $\alpha$ convertase (ADAM17) mediates regulated ectodomain shedding of the severe-acute respiratory syndrome-coronavirus (SARS-CoV) receptor, angiotensin-converting enzyme-2 (ACE2). Journal of Biological Chemistry, 280(34), 30113-30119. Doi: 10.1074/jbc.M505111200

Lechien, J. R., Chiesa-Estomba, C. M., Beckers, E., Mustin, V., Ducarme, M., Journe, F., ... Saussez, S. (2021). Prevalence and 6-month recovery of olfactory dysfunction: a multicentre study of 1363 COVID-19 patients. Journal of Internal Medicine, 290(2), 451-461. Doi: 10.1111/JOIM.13209

Lechien, Jerome R., Chiesa-Estomba, C. M., De Siati, D. R., Horoi, M., Le Bon, S. D., Rodriguez, A., ... Saussez, S. (2020). Olfactory and gustatory dysfunctions as a clinical presentation of mild-to-moderate forms of the coronavirus disease (COVID-19): a multicenter European study. European Archives of Oto-Rhino-Laryngology, 2(0123456789). Doi: 10.1007/s00405020-05965-1

Li, Y. C., Bai, W. Z., \& Hashikawa, T. (2020a). The neuroinvasive potential of SARS-CoV2 may play a role in the respiratory failure of COVID-19 patients. Journal of medical virology, 92(6), 552-555. Doi: 10.1002/ JMV.25728

Li, Y. C., Bai, W. Z., \& Hashikawa, T. (2020b). The neuroinvasive potential of SARS-CoV2 may play a role in the respiratory failure of COVID-19 patients. Journal of Medical Virology, 92(6), 552-555. Doi: 10.1002/ jmv. 25728

Mahboobeh Karimi Galougahi, Jahangir Ghorbani, Mehrdad Bakhshayeshkaram, Ali Safavi Naeini, S. H. (2020). Letters to the Editor Olfactory Bulb Magnetic. (January), 1-2. Doi: 10.1016/j.acra.2020.04.002 
Marinosci, A., Landis, B. N., \& Calmy, A. (2020). Possible link between anosmia and COVID19: sniffing out the truth. European Archives of Oto-Rhino-Laryngology, 19-20. Doi: 10.1007/ s00405-020-05966-0

Netland, J., Meyerholz, D. K., Moore, S., Cassell, M., \& Perlman, S. (2008). Severe Acute Respiratory Syndrome Coronavirus Infection Causes Neuronal Death in the Absence of Encephalitis in Mice Transgenic for Human ACE2. Journal of Virology, 82(15), 7264-7275. Doi: 10.1128/jvi.00737-08

Oliveira, L. M. B., Seminara, S. B., Beranova, M., Hayes, F. J., Valkenburgh, S. B., Schipani, E., ... Vallejo, M. (2001). The Importance of Autosomal Genes in Kallmann Syndrome: Genotype-Phenotype Correlations and Neuroendocrine Characteristics 1. The Journal of Clinical Endocrinology \& Metabolism, 86(4), 1532-1538. Doi: $10.1210 /$ jcem.86.4.7420

Opperman, K., Moseley-Alldredge, M., Yochem, J., Bell, L., Kanayinkal, T., \& Chen, L. (2014). A novel nondevelopmental role of the sax-7/ 11 cam cell adhesion molecule in synaptic regulation in caenorhabditis elegans. Genetics, 199(2), 497-509. Doi: 10.1534/genetics.114.169581

Ren, J., Nie, Y., Lv, M., Shen, S., Tang, R., Xu, Y., ... Wang, T. (2015). Estrogen upregulates MICA/B expression in human non-small cell lung cancer through the regulation of ADAM17. Cellular and Molecular Immunology, 12(6), 768-776. Doi: $10.1038 / \mathrm{cmi} .2014 .101$

Rizzo, P., Vieceli Dalla Sega, F., Fortini, F., Marracino, L., Rapezzi, C., \& Ferrari, R. (2020). COVID-19 in the heart and the lungs: could we "Notch" the inflammatory storm? Basic Research in Cardiology, 115(3), 1-8. Doi: 10.1007/s00395-020-0791-5

Russell, B., Moss, C., Rigg, A., Hopkins, C., Papa, S., \& Van Hemelrijck, M. (2020a). Anosmia and ageusia are emerging as symptoms in patients with COVID-19: What does the current evidence say? Ecancermedicalscience, 14, ed98-ed98. Doi: 10.3332/ ECANCER.2020.ED98

Russell, B., Moss, C., Rigg, A., Hopkins, C., Papa, S., \& Van Hemelrijck, M. (2020b). Anosmia and ageusia are emerging as symptoms in patients with COVID-19: What does the current evidence say? Ecancermedicalscience, 14, 9-10. Doi: 10.3332/ecancer.2020.ed98

Shovlin, S., \& Tropea, D. (2018). Transcriptome level analysis in Rett syndrome using human samples from different tissues. Orphanet Journal of Rare Diseases, 13(1), 1-15. doi: 10.1186/s13023018-0857-8

Smith, T. D., \& Bhatnagar, K. P. (2019). Anatomy of the olfactory system. Handbook of Clinical Neurology, 164, 17-28. Doi: 10.1016/B9780-444-63855-7.00002-2

Stockhorst, U., \& Pietrowsky, R. (2004). Olfactory perception, communication, and the noseto-brain pathway. Physiology and Behavior, 83(1 SPEC. ISS.), 3-11. Doi: 10.1016/j. physbeh.2004.07.018

Sungnak, W., Huang, N., Bécavin, C., Berg, M., Queen, R., Litvinukova, M., ... Figueiredo, F. (2020). SARS-CoV-2 entry factors are highly expressed in nasal epithelial cells together with innate immune genes. Nature Medicine, 26(5), 681-687. Doi: 10.1038/s41591-020-0868-6

Townend, G. S., Ehrhart, F., van Kranen, H. J., Wilkinson, M., Jacobsen, A., Roos, M., ... Curfs, L. M. G. (2018). MECP2 variation in Rett syndrome-An overview of current coverage of genetic and phenotype data within existing databases. Human Mutation, 39(7), 914-924. Doi: 10.1002/humu.23542

Vaira, L. A., Salzano, G., Fois, A. G., Piombino, P., \& De Riu, G. (2020). Potential pathogenesis of ageusia and anosmia in COVID-19 patients. International forum of allergy \& rhinology, 10(9), 1103-1104. Doi: 10.1002/ ALR.22593 
Wang, D., Hu, B., Hu, C., Zhu, F., Liu, X., Zhang, J., ... Peng, Z. (2020). Clinical Characteristics of 138 Hospitalized Patients with 2019 Novel Coronavirus-Infected Pneumonia in Wuhan, China. JAMA - Journal of the American Medical Association, 323(11), 1061-1069. Doi: 10.1001/jama.2020.1585

Wenham, C., Smith, J., \& Morgan, R. (2020). COVID-19: the gendered impacts of the outbreak. The Lancet, 395(10227), 846-848. Doi: 10.1016/S0140-6736(20)30526-2
Yan, C. H., Faraji, F., Prajapati, D. P., Boone, C. E., \& DeConde, A. S. (2020). Association of chemosensory dysfunction and Covid-19 in patients presenting with influenza-like symptoms. International forum of allergy \& rhinology, 1-18. Doi: 10.1002/alr.22579

Zhou, N., Bao, J., \& Ning, Y. (2021). H2V: a database of human genes and proteins that respond to SARS-CoV-2, SARS-CoV, and MERS-CoV infection. BMC Bioinformatics, 22(1). Doi: 10.1186/S12859-020-03935-2 